\title{
Towards a Generic Non-Reflective Characteristic Boundary Condition for Aeroacoustic Simulations
}

\author{
Ryu Fattah, ${ }^{*}$ James Gill, ${ }^{\dagger}$ and Xin Zhang, ${ }^{\ddagger}$ \\ Department of Mechanical and Aerospace Engineering *, \\ The Hong Kong University of Science and Technology, Clear Water Bay, Kowloon, Hong Kong SAR, China. \\ Faculty of Engineering and the Environment, ${ }^{\dagger}$ \\ University of Southampton, Hampshire, SO16 7QF, UK.
}

\begin{abstract}
A blended zonal characteristic boundary condition is proposed following a quantitative investigation of the performance of several non-reflective boundary conditions. Two test cases are considered that investigate the effects of acoustic and vortical plane waves impinging on the domain outflow region. A third test case investigates the effects of broadband turbulent flow impinging on a non-reflective outflow boundary condition. From these studies, two non-reflective boundary conditions based on a zonal characteristic method are found to provide a minimal acoustic response for impinging acoustic and vortical disturbances, respectively. These methods both make use of the transverse characteristic terms to improve performance, although each method uses a different inclusion of these terms. A final boundary condition is proposed that blends the performance of the two zonal characteristic methods. A blending function is used that switches the characteristic boundary condition smoothly between regions dominated by acoustic, or vortical, disturbances. The feasibility of this novel method is demonstrated on a test case where broadband turbulence impinges on a small section of an outflow region.
\end{abstract}

Keywords: computational aeroacoustics, non-reflective boundary conditions

\section{Introduction}

$T_{\text {HERE is a requirement in computational aeroacoustic (CAA) simulations for effective non-reflecting }}$

1 boundary conditions at the outer edges of a computational domain. A computational domain is usually truncated, which combined with the use of numerical schemes that are optimized for minimal dispersion and dissipation errors, makes simulations sensitive to spurious acoustic reflections from acoustic and vortical waves impinging on the domain boundaries. A variety of non-reflective boundary conditions have been developed for the Navier-Stokes (N-S), Euler, and Linearised Euler equations (LEE). The key methods in achieving minimal reflections along domain boundaries are: asymptotic far-field solutions, buffer-zone techniques, and characteristic methods.

The asymptotic far-field condition by Tam and Dong ${ }^{1}$ is a generalised version of the radiation condition by Tam and Webb. ${ }^{2}$ This boundary condition, derived from the linearised Euler equations, assumes that acoustic waves propagate from a point source in the far-field, and that vorticity and entropy waves are convected by the mean flow. This method was extended to three-dimensional cases by Bogey and Bailly. ${ }^{3}$ The implementation requires prior knowledge of the mean flow, which is not always readily available. Additionally, pressure variations are assumed to be entirely acoustic. This weakens the non-reflective performance from outgoing

*Research Fellow,

$\dagger$ Research Fellow, Airbus Noise Technology Centre.

$\ddagger$ Swire Professor of Aerospace Engineering, Associate Fellow, AIAA. aexzhang@ust.hk. 
vortical waves that contain a significant pressure distribution. ${ }^{3}$ However, this issue may be overcome by the application of a buffer-zone to damp vorticity fluctuations before they reach the outflow face. Finally, the radiation condition should be applied across a region in order to be compatible with non-linear governing equations. $^{3}$

Buffer-zone techniques ${ }^{4}$ force the flow variables to a target value. This methodology aims to prevent acoustic reflections by damping the outgoing disturbances, via a buffer-zone (or a sponge-zone), before they impinge on the outflow face. The solution in the buffer region is non-physical, and is therefore applied as an extension of the original computational domain. The numerical reflections generated within the damping region are affected by tuneable parameters that define dimensions of the buffer-zone, and the shape of the damping function. ${ }^{5,6} \mathrm{~A}$ variant of the buffer-zone method known as the perfectly matched layer ${ }^{7}$ is a technique that overcomes the issue of reflections generated by the damping process. This method can provide good results but is difficult to apply to the N-S equations. ${ }^{8}$

Non-reflective boundary conditions based on a characteristic analysis of the governing equations are commonly applied in compressible flows. Thompson ${ }^{9,10}$ proposed a characteristic boundary condition (CBC) for the Euler equations, and Poinsot and Lele ${ }^{11}$ extended this method to the N-S equations. The CBCs assume a one-dimensional flow in the direction normal to the outflow boundary via the Locally One Dimensional Inviscid (LODI) relations. The amplitudes of the reflected characteristic waves are modified to ensure minimal reflections and a well-posed system. Some authors, such as Yoo and $\mathrm{Im}^{12}$ have extended this method to multi-dimensional problems by including a part of the transverse source terms in the boundary condition. Low Mach number analysis shows that the contribution of the transverse terms should scale with the reference Mach number. ${ }^{12}$ However, there are open questions about the definition of the reference Mach number when the outflow region is inhomogeneous. ${ }^{8}$ Liu and Vasilyev ${ }^{13}$ argue that the contribution by particular transverse terms should be included in the boundary condition, and additionally that this contribution should depend on the wave angle of the disturbance crossing the boundary. Sandberg and Sandham ${ }^{14}$ proposed a zonal CBC where the amplitude of the reflected characteristic wave is gradually damped through a buffer region, which significantly reduces the reflections cause by outgoing vortical waves. This method is different to a standard buffer-zone method where damping is applied to the primitive variables, and recently Gill et al. proposed a zonal CBC that includes the transverse terms. ${ }^{6}$

Previous studies ${ }^{3,5,15}$ have compared the performance of different non-reflective boundary conditions. Generally, it is found that the far-field method is ideal for purely acoustic problems, ${ }^{8}$ and that the characteristic methods provide better results for cases that additionally contain vortical waves. ${ }^{8}$ Despite the various methods that have been developed, no method currently exists to remove all reflections for all cases. Factors such as the frequency, mean flow non-uniformity, wave angle, and Mach number, can affect the performance. ${ }^{5}$

Recently, Gill et al. ${ }^{6}$ developed a new, generic zonal characteristic method and optimized the variable parameters to minimize the acoustic reflections generated purely by incoming acoustic disturbances. The new condition was compared quantitatively against several other types of non-reflective boundary condition and was found to perform consistently well across a wide range of angular frequency, Mach number $M$, and cut-on ratio $\xi$, defined by the ratio of streamwise to transverse wave numbers $k_{x} / k_{y}$. Additionally, an optimization of the tuneable parameters for the implicit buffer-zone method ${ }^{6}$ was also presented.

The aim of this paper is to extend the work by Gill et al. ${ }^{6}$ by enhancing the zonal characteristic boundary condition to be non-reflective for incoming vortical disturbances. The acoustic plane wave study used previously by Gill et al., ${ }^{6}$ and two new studies aimed at quantifying the acoustic response from vortical disturbances, are applied in the present work. The combined outcomes from these works aim to develop a generic non-reflective boundary condition for CAA, based on the zonal characteristic method.

This paper is organized as follows. In Section II the numerical methods that are applied to the governing equations, and the evaluated boundary conditions, are given. Three test cases are then considered. In Section III, an acoustic plane wave test ${ }^{5,6}$ is used to identify the ideal method for problems consisting only of acoustic disturbances. In Section IV.A a similar test case, applying plane vortical waves instead of plane acoustic waves, is considered to highlight the most suitable boundary condition for problems consisting of only vortical plane wave disturbances. In Section IV.B the vortical plane wave study is extended to a more realistic test case. In this test a synthetic, two-dimensional, frozen, and isotropic turbulence impinges on a portion of the outflow region. In Section V, an extension to the optimized zonal CBC by Gill et al. ${ }^{6}$ is proposed and tested on the latter test case, that switches to another CBC in regions of the outflow dominated by vortical disturbances. 


\section{Numerical Methods}

In this work, a high-order solver previously used to study linear ${ }^{5,6,16}$ and non-linear ${ }^{17,18}$ CAA problems, is used to perform the numerical simulations.

\section{II.A. Governing Equations}

The two-dimensional compressible Euler equations, in full conservation form, are solved here. The governing equations are expressed as,

$$
\frac{\partial \boldsymbol{Q}}{\partial t}+\frac{\partial \boldsymbol{E}}{\partial x}+\frac{\partial \boldsymbol{F}}{\partial y}=0
$$

where the conserved variable vector $\boldsymbol{Q}$, and the fluxes in the stream-wise $\boldsymbol{E}$, and transverse $\boldsymbol{F}$, directions are given by,

$$
\boldsymbol{Q}=\left(\begin{array}{c}
\rho \\
\rho u \\
\rho v \\
\rho e_{T}
\end{array}\right), \quad \boldsymbol{E}=\left(\begin{array}{c}
\rho u \\
\rho u^{2}+p \\
\rho u v \\
\rho\left(e_{T}+p\right) u
\end{array}\right), \quad \boldsymbol{F}=\left(\begin{array}{c}
\rho v \\
\rho u v \\
\rho v^{2}+p \\
\rho\left(e_{T}+p\right) v
\end{array}\right)
$$

where $\rho$, is the density, $u$ and $v$ are the velocity components in the stream-wise and transverse directions, and $p$ is the static pressure. The free-stream quantities for the density and speed of sound, are used in combination with a reference length to express the governing equations in a non-dimensional form. The total energy $e_{t}$ per unit mass is defined by,

$$
e_{T}=\frac{p}{\rho(\gamma-1)}+\frac{1}{2} u_{i} u_{i}
$$

where $\gamma=c_{p} / c_{v}$ is the ratio of specific heats. An initialized flow, defined across a curvilinear structured grid, is marched in time by a fourth-order Runge-Kutta scheme ${ }^{7}$ using a maximum Courant number of 0.4. The flux terms are evaluated by fourth-order accurate spatial schemes ${ }^{19,20}$ and the solution is filtered by sixth-order implicit filters ${ }^{21}$ at the end of each time-step.

\section{II.B. Buffer-zone Technique}

The explicit buffer-zone technique applies a forcing to the primitive variables at the end of each Runge-Kutta stage by,

$$
\hat{\boldsymbol{U}}^{n+1}=\boldsymbol{U}^{n+1}-\sigma(x)\left(\boldsymbol{U}^{n+1}-\boldsymbol{U}_{T}\right)
$$

where $\boldsymbol{U}^{n+1}$ is the primitive variable vector at the end of each time step, $\boldsymbol{U}_{T}$ is the vector containing the prescribed target values, $\hat{\boldsymbol{U}}^{n+1}$ is the vector after the buffer-zone treatment, and $\sigma(x)$ is the damping function defined as,

$$
\sigma(x)=\alpha\left(1-\frac{L-x^{*}}{L}\right)^{\beta},
$$

where $L$ is the length of the buffer-zone, $x^{*}$ is the distance from the inner boundary of the buffer-zone, and the parameters $\alpha$ and $\beta$ define the damping function. In this work, the coefficient $\alpha$ is scaled by the time step of the Runge-Kutta stage to perform as an implicit buffer-zone i.e., $\alpha^{*}=\alpha \Delta t$. The coefficients $\alpha=6$, $\beta=2$ define the implicit buffer-zone (IBZ) method optimized for acoustic disturbances. ${ }^{6}$

\section{II.C. Characteristic Outflow}

The governing equation given by Equation 1 can be rewritten as,

$$
\frac{\partial \boldsymbol{Q}}{\partial t}+\boldsymbol{A} \frac{\partial \boldsymbol{Q}}{\partial x}=-\boldsymbol{B} \frac{\partial \boldsymbol{Q}}{\partial y}
$$


where $\boldsymbol{A}$ and $\boldsymbol{B}$ are the Jacobian matrices of the flux derivatives, and $\boldsymbol{Q}$ is the conserved variable vector. This formulation considers the $y$-derivatives as a source term. By diagonalizing the flux Jacobian matrix $\boldsymbol{A}$, this system can be expressed by its characteristic waves along the $x$-direction. The eigenvalues of this system are defined by the diagonal terms of $\boldsymbol{\Lambda}$, and it is related to the flux Jacobian $\boldsymbol{A}$ by the matrix $\boldsymbol{P}$ and its inverse $\boldsymbol{P}^{-1}$ by, $\boldsymbol{\Lambda}=\boldsymbol{P}^{-1} \boldsymbol{A P}$. Therefore, Equation 1 can be expressed in the following characteristic form,

$$
\frac{\partial \boldsymbol{R}}{\partial t}+\boldsymbol{\Lambda} \frac{\partial \boldsymbol{R}}{\partial x}=\mathcal{T}, \quad \partial \boldsymbol{R}=\boldsymbol{P}^{-1} \boldsymbol{Q}
$$

The characteristics $(\mathcal{L})$ are defined as,

$$
\mathcal{L}=\boldsymbol{\Lambda} \frac{\partial \boldsymbol{R}}{\partial x}
$$

and the source terms on the right hand side are typically decomposed into viscous $\left(\mathcal{S}_{v}\right)$ and transverse $(\mathcal{T})$ terms. In the Euler equations the viscous effects are neglected. For subsonic outflows, the characteristic boundary condition is applied to $\mathcal{L}_{5}$, which corresponds to the eigenvalue $u-c$, and it corrects the flux derivatives. Zero acoustic reflections from an outflow region may be obtained by setting $\mathcal{L}_{5}=0$. However, this condition may result in a drifting mean pressure. ${ }^{11}$ A generalized outflow boundary condition that overcomes this problem is defined by,

$$
\mathcal{L}_{5}=K\left(p-p_{T}\right)+A \mathcal{T}_{5}, \quad K=\sigma\left(1-M^{2}\right)(c / l),
$$

where $p_{T}$ is a target pressure, $\mathcal{T}_{5}$ represents the transverse terms, and $K$ is a constant defined in terms of a characteristic length $(l)$, the Mach number $(M)$, the sound speed $(c)$, and a weighting parameter $(0.1<\sigma<0.4) .{ }^{11}$ The definition of $A$ in Equation 9 controls the strength of the transverse terms. A characteristic boundary condition may be implemented locally only along an outflow face or across a zone. In the latter, the characteristic variables are ramped towards a target value, similar to a buffer-zone method.

\section{II.D. Blended characteristic outflow}

An outflow region may be subjected primarily to acoustic disturbances, and additionally by a local region of strong vortical disturbances (for example the wake behind a bluff body). In this circumstance, different zonal characteristic methods may best suited for the two regions dominated by different types of disturbances. A proposed blending function $s_{f}$ is implemented into the characteristic boundary condition by,

$$
\mathcal{L}_{5}=s_{f} \mathcal{L}_{5}^{A}+\left(1-s_{f}\right) \mathcal{L}_{5}^{B},
$$

which switches between two characteristic boundary conditions, denoted by $\mathcal{L}_{5}^{A}$ and $\mathcal{L}_{5}^{B}$. Further details to this proposed method are provided together with a demonstration later in Section V.

\section{II.E. Evaluated boundary conditions}

A summary of the boundary conditions evaluated in this work is given in Table II.E. The first is an optimized implicit buffer zone (IBZ) method. ${ }^{6}$ The second is a zonal $\mathrm{CBC}^{14}$ that assumes a one-dimensional flow (ZODI). The third is a zonal CBC that considers all of the transverse terms (ZTCBC). The fourth is the local CBC method proposed by Yoo and $\operatorname{Im}^{12}$ (LTCBC-M), and the fifth is a zonal extension of Yoo and Im's method used by Gill et al. ${ }^{6}$ (ZTCBC-M). The sixth is the optimized zonal CBC method by Gill et al. ${ }^{6}$ (ZTCBC-O) that is also evaluated in this work. In Section V, a novel blended method (ZTCBC-B) that combines the ZTCBC-M and ZTCBC-O methods is proposed and tested.

\section{Tests on Acoustic Plane Waves}

The acoustic plane wave test case, previously used by Gill et al., ${ }^{6}$ is illustrated by Figure 1 . In this test case an acoustic plane wave of specific streamwise $\left(k_{x}\right)$ and transverse $\left(k_{y}\right)$ wave number is introduced by an implicit inflow buffer-zone method, and propagated towards the outflow boundary. The ratio of the wave numbers defines the cut-on ratio $\xi=k_{x} / k_{y}$, and the reflected pressure waves are isolated from the solution 


\begin{tabular}{lll}
\hline Name & Description & Parameters \\
\hline IBZ & Implicit Buffer Zone $^{6}$ & $\alpha=6, \beta=2$ \\
ZODI & Zonal One Dimensional Inviscid relation $^{14}$ & $A=0$ \\
ZTCBC & Zonal Transverse CBC with unit scaling & $A=1$ \\
LTCBC-M & Local Transverse CBC with Mach number scaling ${ }^{12}$ & $A=1-M$ \\
ZTCBC-M & Zonal Transverse CBC with Mach number scaling $^{6}$ & $A=1-M$ \\
ZTCBC-O & Zonal Transverse CBC with optimized parameters $^{6}$ & $A=\max (0.0,(0.3-0.7 M))$ \\
ZTCBC-B & Proposed blending of ZTCBC-M and -O methods $^{\text {Z }}$ & Equation 10 \\
\hline
\end{tabular}

Table 1. Overview of the boundary conditions for evaluation.

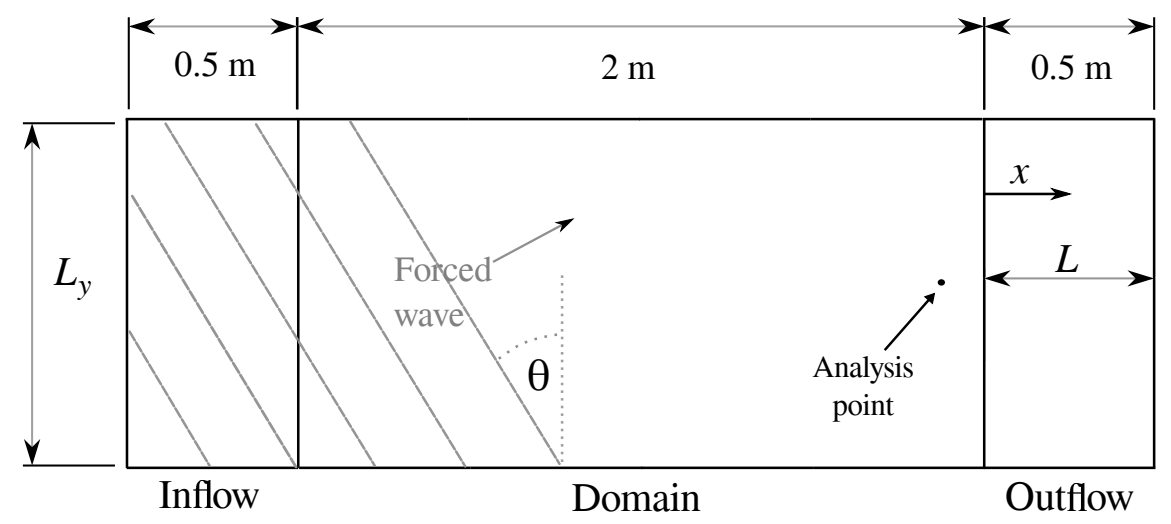

Figure 1. Schematic of the acoustic plane wave study used previously by Gill et al. ${ }^{6}$

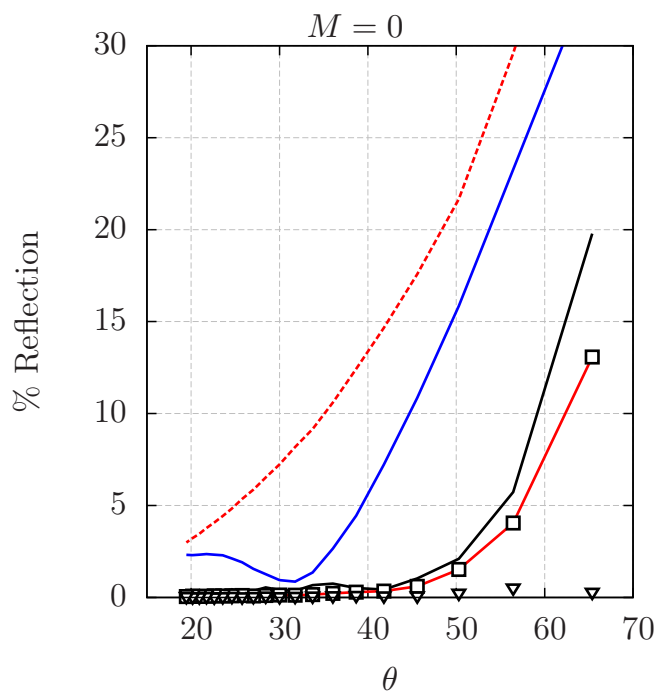

IBZ ZODI

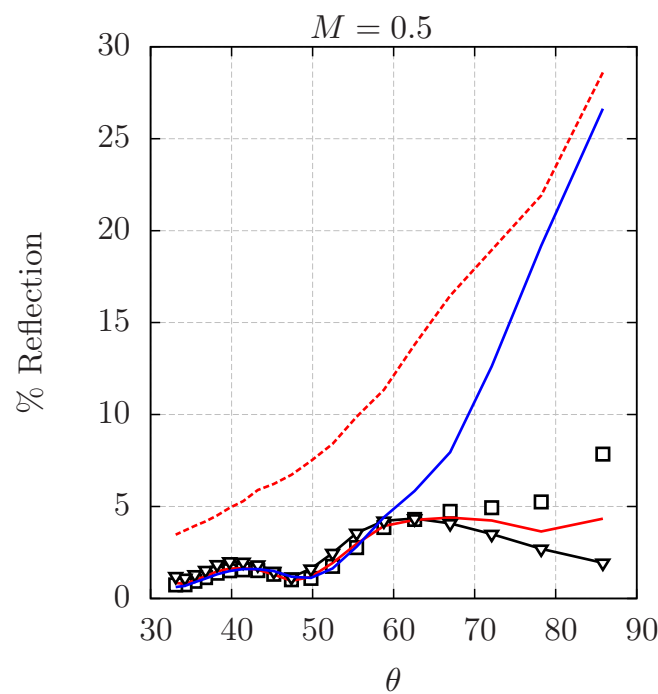

ZTCBC $\square$

Figure 2. The spurious reflections generated by an acoustic wave impinging an outflow boundary at $M=0$ (left), and $M=0.5$ (right).

field by a wave splitting technique. ${ }^{22}$ The reflection is defined by the ratio of sound pressure levels of the reflected left-travelling acoustic wave, and the right-travelling acoustic waves.

At a Mach number of $M=0$, the CBCs defined by ZTCBC and ZTCBC-M are equivalent, and at 
a Mach number of $M=0.5$, the ZTCBC-O is equivalent to ZODI. The results from the acoustic plane wave tests, shown in Figure 2, highlight three key points. Firstly, the ZTCBC-O method provides the best performance across the range of wave angles and generates a maximum reflection of approximately $4-5 \%$ at a Mach number of $M=0.5$ and a wave angle of 60 degrees. Secondly, the acoustic reflections generated by the LTCBC-M ${ }^{12}$ method can be reduced significantly by applying the method across a zone (ZTCBC-M). However, the performance of the ZTCBC-M deteriorates as the wave angle exceeds 50 degrees. Finally, all the zonal CBC methods perform better than the optimized IBZ method. However, the IBZ performs well for wave angles below 40 degrees, with reflections of less than $5 \%$.

\section{Tests on Vortical Waves}

In this section, two test cases are considered that quantify the acoustic response due to vortical disturbances impinging on the outflow boundary conditions listed in Table II.E. Firstly, a study of vortical plane waves impinging on an entire outflow face is presented. Secondly, a study of a synthetic turbulent field impinging on a smaller section of the entire outflow boundary is shown.

\section{IV.A. Vortical plane wave}

A single-frequency vortical plane wave is imposed across the inflow region as shown by Figure 3, where the following conditions are applied,

$$
z(x, y, t)=k_{x}(x-M t)+k_{y} y, \quad u^{\prime}(x, y, t)=-\epsilon \cos (z) \frac{k_{y}}{\sqrt{k_{x}^{2}+k_{y}^{2}}}, \quad v^{\prime}(x, y, t)=\epsilon \cos (z) \frac{k_{x}}{\sqrt{k_{x}^{2}+k_{y}^{2}}}
$$

where $z$ is an auxiliary equation, $k_{x}$ and $k_{y}$ are the streamwise and transverse wave numbers, $M$ is the free-stream Mach number, $x$ and $y$ are the spatial coordinates, $t$ is the time, and $\epsilon$ is the gust amplitude. The disturbance is divergence-free and does not generate acoustic waves. When these vortical waves pass through an outflow region, they may be deformed. The deformation violates the divergence-free condition and can generate upstream and downstream acoustic waves. The downstream traveling wave may interact with the outflow region and form a secondary acoustic reflection that may also propagate upstream.

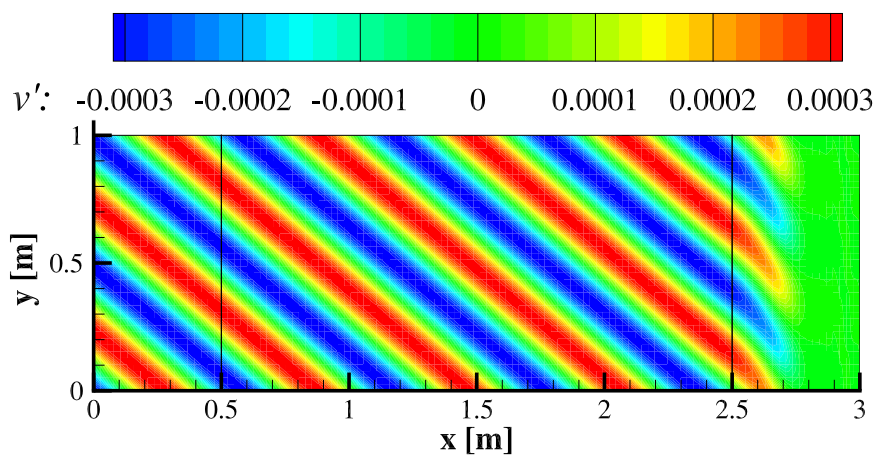

Figure 3. Plane vortical gust test using an implicit buffer zone (IBZ) outflow boundary condition.

The performance of the outflow boundary condition is evaluated by a non-dimensional reflection coefficient $(R)$ defined as,

$$
R=\frac{p_{r m s}}{0.5 \rho_{\infty}\left(u_{r m s}^{2}+v_{r m s}^{2}\right)},
$$

where $p_{r m s}$ is a measure of the acoustic energy recorded near the outflow region at $(x, y)=(2,0.5) \mathrm{m}$, and the denominator is a reference measure of the vortical gust kinetic energy, recorded in the inflow region at $(x, y)=(0,0.5) \mathrm{m}$. 
Generally, the length of the zonal/buffer region should be based on the largest wavelength, and at low Mach numbers the wavelength of the acoustic mode is sufficiently larger than that of the vortical mode (at a fixed angular frequency). Therefore, a typical zonal/buffer region length $(L)$ will tend to be larger than the vortical disturbance wavelength $\left(\lambda_{x}\right)$ i.e., $\lambda_{x} / L<1$. The results from a variety of individual test cases all at $\lambda_{x} / L=0.5$ and various gust angles are plotted in Figure 4 , and the following observations are made. At a Mach number of $M=0.2$ and $M=0.5$, the IBZ method tends to generate the largest reflections. The ZODI and ZTCBC methods provide a slight improvement over the IBZ. The performance of the more advanced LTCBC-M can be improved towards the low wave angle range when it is implemented across zone (ZTCBC-M). Finally, the optimized ZTCBC-O method does not perform as well for vortical plane waves as it does for acoustic plane waves.

The IBZ method appears to be the least suitable method for vortical outflows, as it directly modifies the primitive variables. This method may significantly affect the divergence-free condition across the bufferregion and generate spurious acoustic waves that travel both upstream and downstream. Characteristic methods aim to overcome this issue by modifying the amplitude of the upstream traveling acoustic wave, while leaving the downstream traveling vortical wave unperturbed. However, at lower Mach numbers and higher gust angles, the importance of the transverse term becomes greater. ${ }^{12}$ This explains the wider variance by different CBC methods at lower Mach number, and the reduced performance by CBCs that do not account for the transverse terms correctly.

\section{IV.B. Broadband turbulence}

A synthetic turbulent spectra is generated by an advanced digital filter method. ${ }^{16}$ Digital filter methods have been extensively used for CAA applications ${ }^{23,24,25}$ due to their ability to reproduce realistic turbulence spectra with a low computational cost. In the current work, an advanced digital filter method ${ }^{16}$ is used to generate a two-dimensional, isotropic, and frozen turbulence with a Gaussian energy spectrum, which is specified by the integral length scale $\Lambda$, and the turbulent intensity $I_{x x}$. The test case is illustrated in Figure 5 and it consists of nine blocks. Each block consists of a uniformly spaced grid with $\Delta x=\Delta y=0.02 \mathrm{~m}$. Periodic boundary conditions are applied to the lower and upper bounds of the domain. The inflow region is set as a pressure far-field condition, and the outflow region is set to one of the methods listed in Table II.E. However, the LTCBC-M will be dismissed for the remainder of the work, as the zonal implementation (ZTCBC-M) has performed better for both acoustic and vortical plane waves.

In this test case, illustrated by Figure 5 , the turbulent flow is convected along the $x$-axis by the free-stream Mach number $(M)$, and the impingement angle $(\alpha)$ can be varied by rotating the grid about $(x, y)=(3 \mathrm{~m}, 3 \mathrm{~m})$. The synthetic turbulence is introduced across a region defined by $x=2 \mathrm{~m}$ and $2.25 \mathrm{~m}<y<3.75 \mathrm{~m}$, and the time history of the induced velocity field is recorded at the center of the domain. A secondary monitor is placed at the center of the top left block, which lies outside the turbulent region and is used to quantify the acoustic reflections. The effect of free-stream Mach number $(M)$, the length of the zonal/buffer region $(L)$, impingement angle $(\alpha)$, and the integral length scale $(\Lambda)$ are investigated for each outflow boundary condition. The turbulence intensity and zonal/buffer region length are set to $I_{x x}=0.01 U_{\infty}$ and $L=0.4 \mathrm{~m}$, respectively, unless stated otherwise.

The cumulative velocity energy spectra for varying Mach number, impingement angle, and turbulent integral length scales are plotted in Figure 6. This figure shows that a frequency axis normalized by the integral length scale $\Lambda$ and by the free-stream velocity $U_{\infty}$ will give a good collapse of spectra, and therefore shows that $f \Lambda / U_{\infty}$ is suitable to identify which parameters have the greatest effect on the acoustic response.

An overall comparison of the acoustic response generated by broadband turbulence impinging on various outflow boundary conditions is given by Figure 7. The following key observations highlight a similar trend found from the vortical plane wave study in Section IV.A. Firstly, the IBZ method performs poorly compared to the characteristic methods. Secondly, the ZTCBC-M method generates the lowest acoustic response across the frequency range shown. Finally, the optimized ZTCBC-O does not perform as well for vortical waves as it does for acoustic waves.

The changes to the performance of each boundary condition due to varying Mach number, integral length scale, zonal/buffer region length, and impingement angle are illustrated by Figures 8, 9, 10, and 11, respectively. The lowest acoustic response for any test case is consistently obtained by the ZTCBCM method. The ZTCBC-M method provides similar performance with various integral length scales and zonal/buffer region lengths. It is however, quite sensitive to the Mach number. In Figure 8, an increase of $60 \mathrm{~dB}$ across all frequencies is observed. This may indicate a limitation of the low Mach number analysis ${ }^{12}$ 


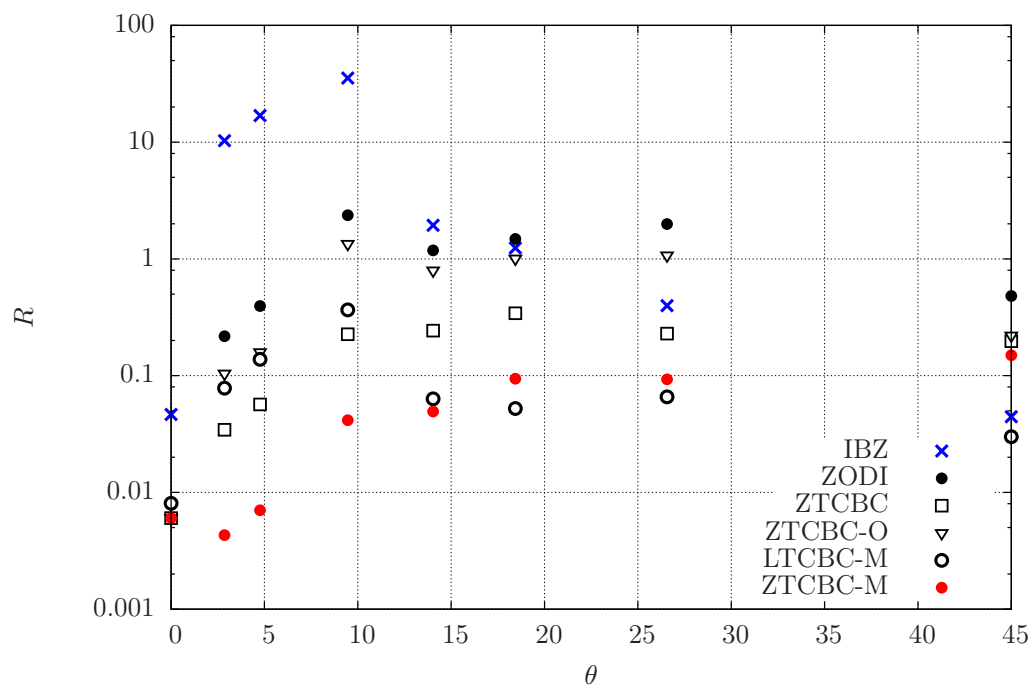

(a) $M=0.2$

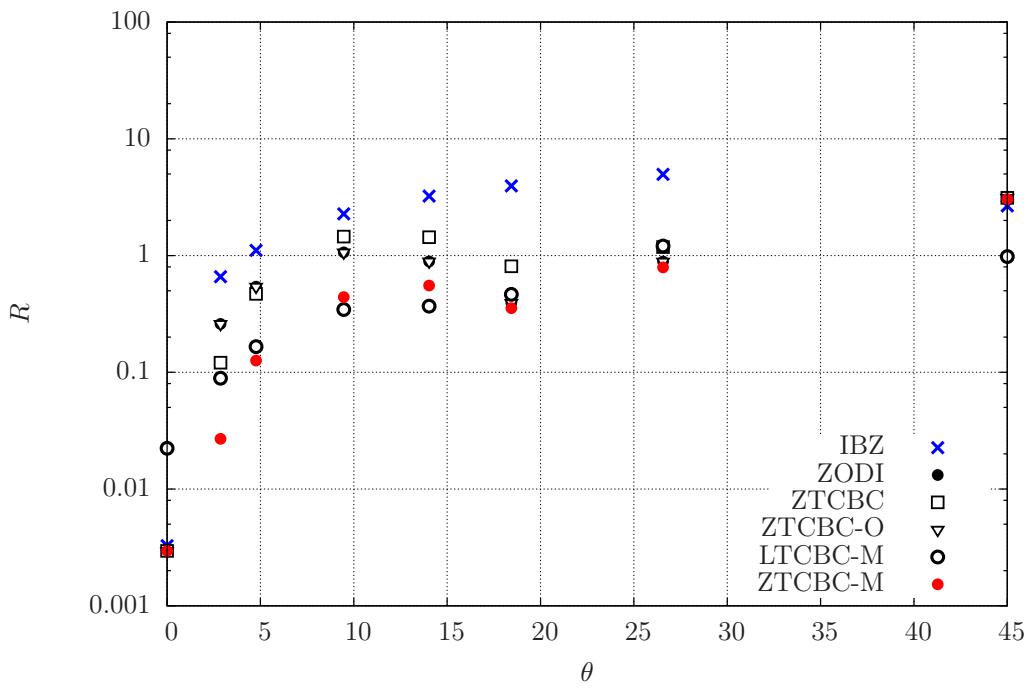

(b) $M=0.5$

Figure 4. Acoustic reflection by vortical plane waves at various gust angles $\theta$ impinging on various outflow boundary conditions.

used to derive the transverse term weighting used in the ZTCBC-M method. The high sensitivity to Mach number is additionally demonstrated by varying the impingement angle. In this test case the grid was rotated, but the reference Mach number used in the $\mathrm{CBC}$ remained unchanged. Therefore, the changes to the spectrum illustrated in Figure 11, are due to an improper definition of the reference Mach number. As the grid is rotated further, the Mach number normal to the outflow boundary is reduced. The net effect is an over-prediction of the transverse term weighting in the ZTCBC-M method, resulting in an acoustic response behavior that matches more closely to the results obtained by the ZTCBC.

The other boundary conditions (not including the ZTCBC-M), are highly sensitive to the zonal/buffer region length $(L)$. As the length is increased, the acoustic response spectrum tends to lower levels, and converges to the spectrum obtained from the ZTCBC-M method (see Figure 10). The sensitivity of the IBZ method to varying Mach number and the integral length scale, may simply be due to the effective changes to the zonal/buffer region length. A lower Mach number will increase the exposure time of the vortices to the damping in the buffer-region, and a smaller integral length scale will form a velocity spectra that is focused 


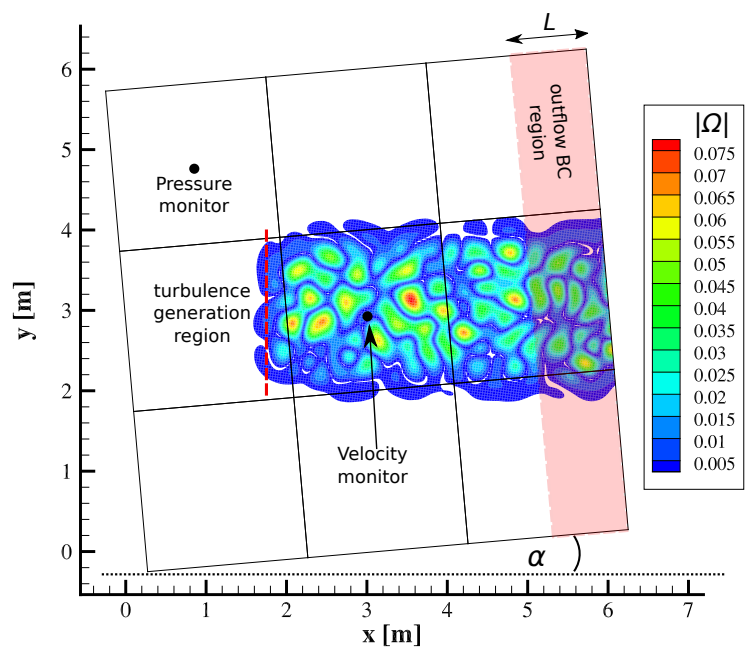

Figure 5. Schematic of the test case with a synthetic turbulent flow highlighted by the vorticity magnitude.

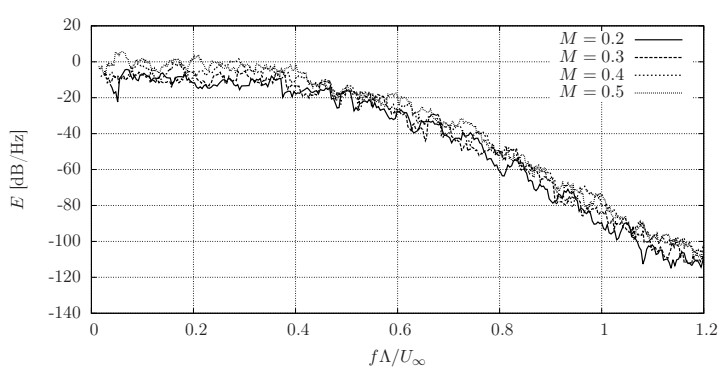

(a) Varying $M$

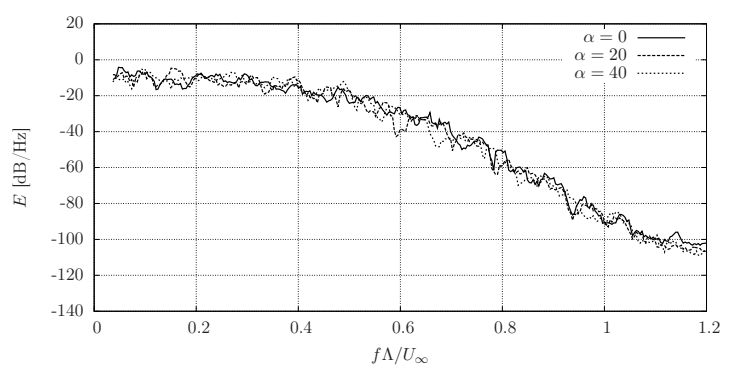

(b) Varying $\alpha$

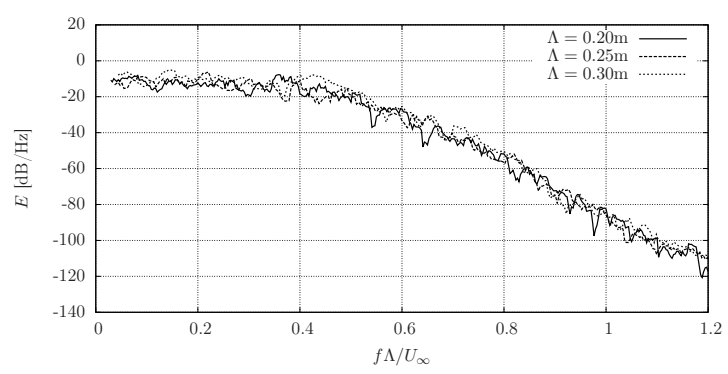

(c) Varying $\Lambda$

Figure 6. Velocity energy spectra normalized by the turbulent intensity. 


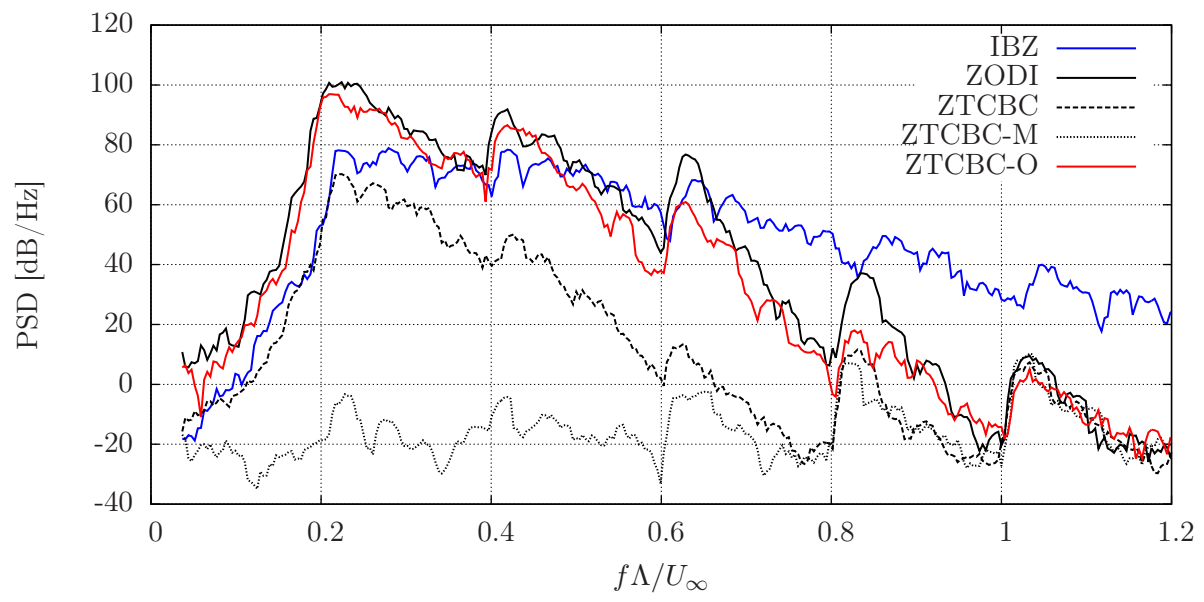

Figure 7. The acoustic pressure response by synthesized turbulence using $\Lambda=0.25 \mathrm{~m}$, at a $\mathrm{Mach}$ number of $M=0.2$.

towards smaller length scales, thereby decreasing the ratio $\lambda_{x} / L$. An increased buffer-zone length can also be induced by a highly rotated grid. The overall performance of the IBZ is not as good as the CBC methods, as the boundary condition induces significant vortex deformation. For the CBC, the trends in the acoustic response spectrum are additionally sensitive to the contribution of the transverse term, and the best results are obtained from the ZTCBC-M method. 


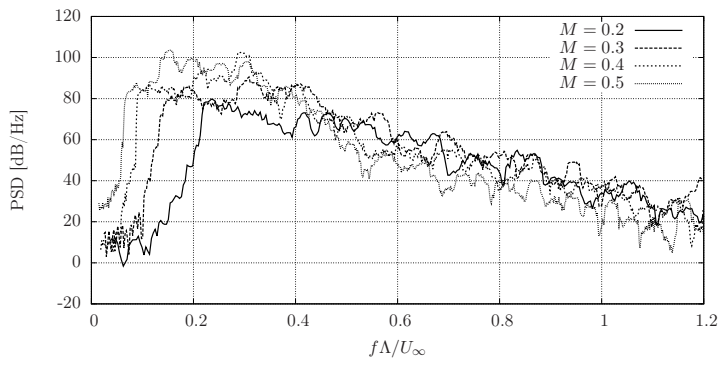

(a) IBZ

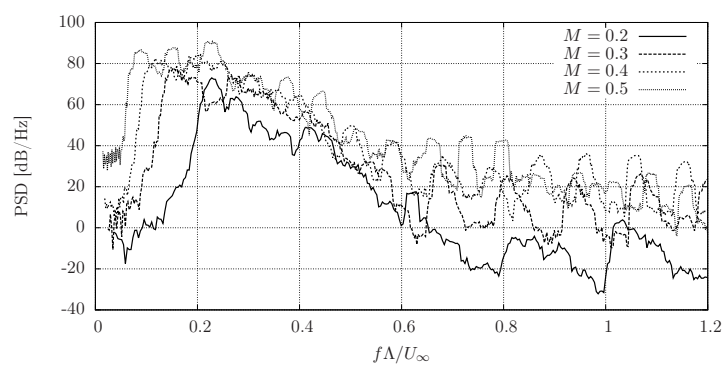

(c) ZTCBC

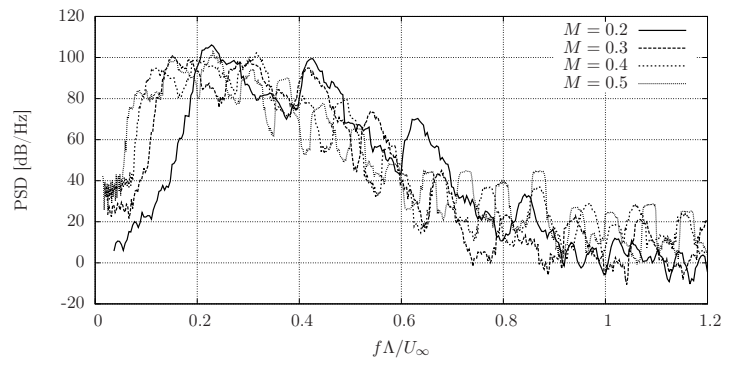

(b) ZODI

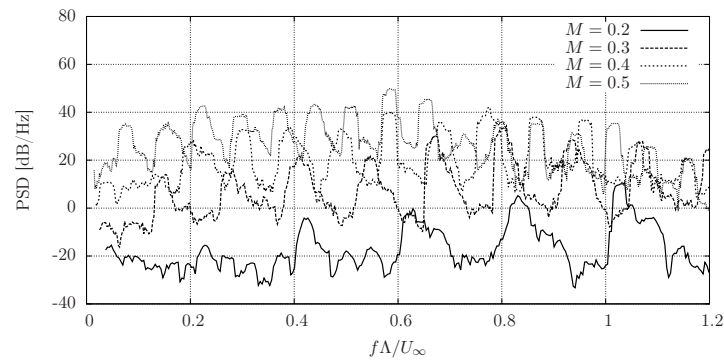

(d) ZTCBC-M

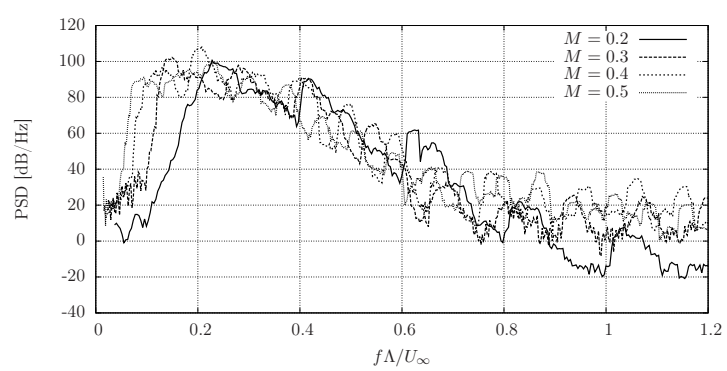

(e) ZTCBC-O

Figure 8. The acoustic responses by synthesized turbulence at varying Mach number. 


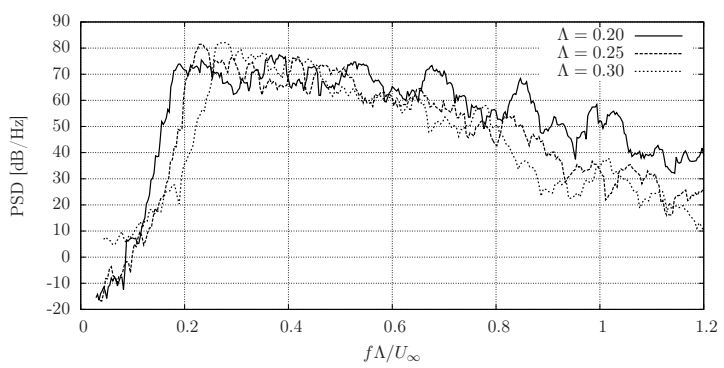

(a) IBZ

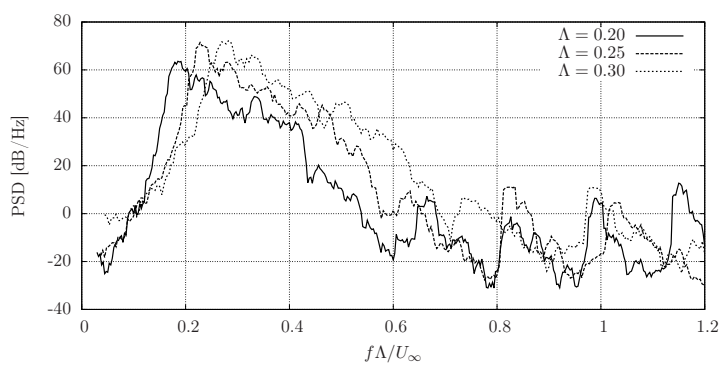

(c) ZTCBC

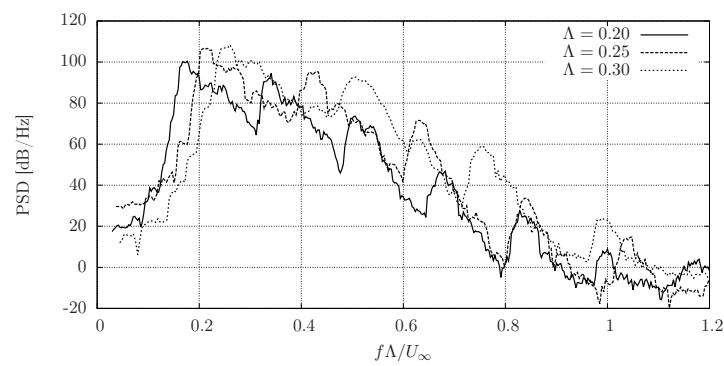

(b) ZODI

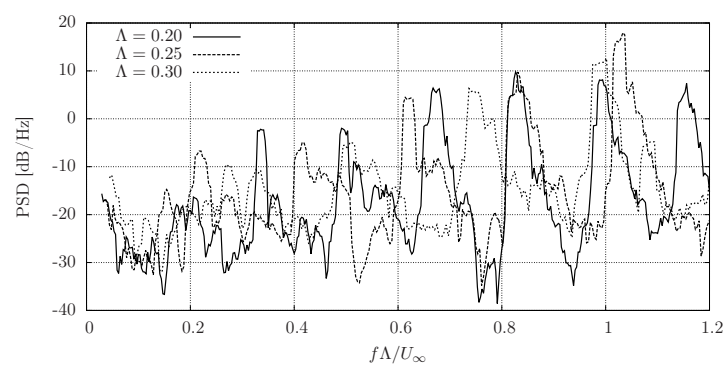

(d) ZTCBC-M

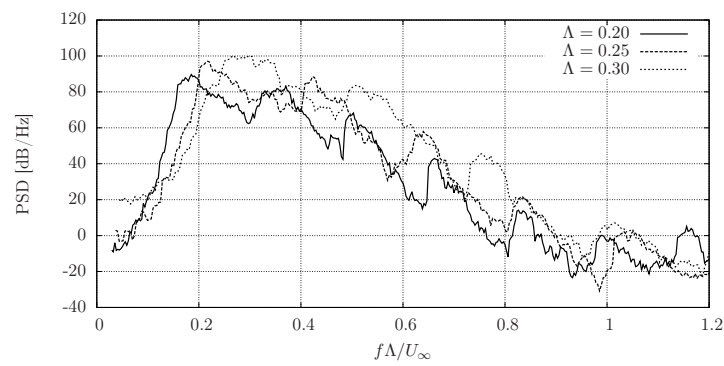

(e) ZTCBC-O

Figure 9. The acoustic responses by synthesized turbulence with varying integral length scale $\Lambda$, at a fixed Mach number of $M=0.2$. 


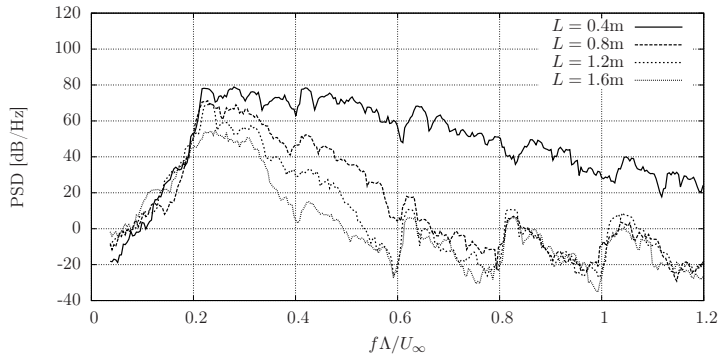

(a) IBZ

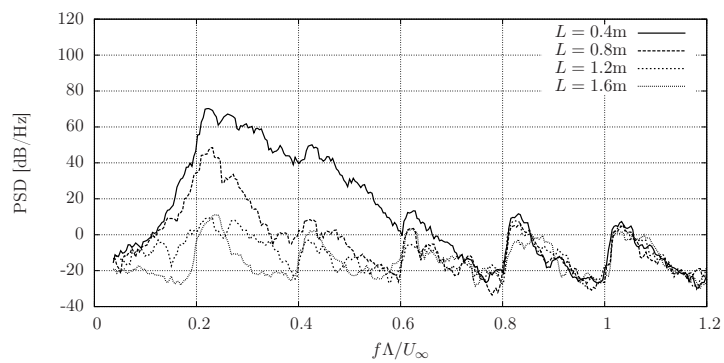

(c) ZTCBC

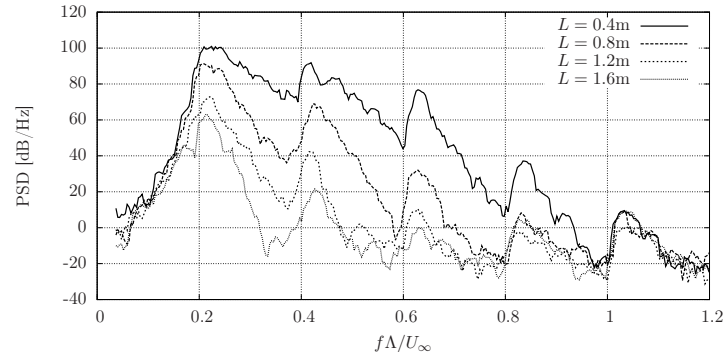

(b) ZODI

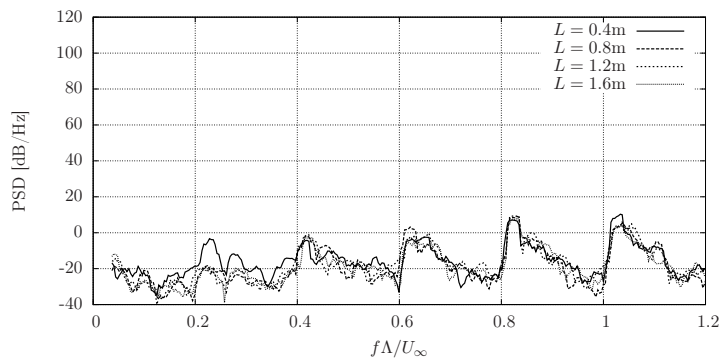

(d) ZTCBC-M

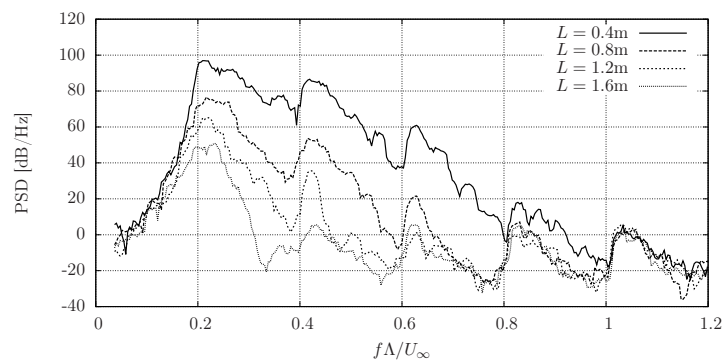

(e) ZTCBC-O

Figure 10. The acoustic responses by synthesized turbulence with varying zonal/buffer region lengths $L$.

\section{Blended Zonal Boundary Condition}

From the acoustic test case in Section III, and the vortical test cases in Sections IV.A and IV.B, it has been shown that different ZTCBC methods are best suited for different types of outflow boundary. Outflow boundaries whose flow is dominated by acoustic, or vortical, disturbances generate the smallest acoustic response by the ZTCBC-O and ZTCBC-M methods, respectively. In this Section, a blended outflow boundary condition (ZTCBC-B) is proposed that combines the ZTCBC-O and ZTCBC-M methods such that the ZTCTB-O method is used for outflows dominated by acoustic waves and the ZTCBC-M is used when strong vortical waves are present. The blending is simple to implement, since it involves only a change in the weighting of the transverse characteristic terms. The feasibility of the blended outflow boundary condition (ZTCBC-B) is tested on a case where broadband turbulence impinges on a small region of the outflow. A blending scalar function $\left(s_{f}\right)$ that ranges from 0 to 1 is defined by:

$$
s_{f}(y)=\frac{1}{2} \min \left(1+\tanh \left(\frac{y-\left(y_{c}-\phi\right)}{d}\right), 1-\tanh \left(\frac{y-\left(y_{c}+\phi\right)}{d}\right)\right),
$$

where $y_{c}$ is the centerline position of the synthetic eddy injection plane, and $\phi$ defines the distance away from $y_{c}$ at which the blending function switches from $s_{f}=1$ to $s_{f}=0$, and $d$ specifies the distance over which the blending function varies. The blending scalar is applied to the characteristic outflow boundary condition by Equation 10. When $s_{f}=1$, the ZTCBC-M method is applied, and when $s_{f}=0$, the ZTCBC-O 


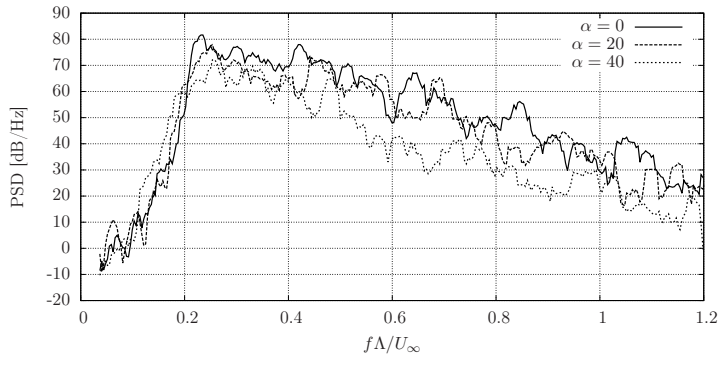

(a) IBZ

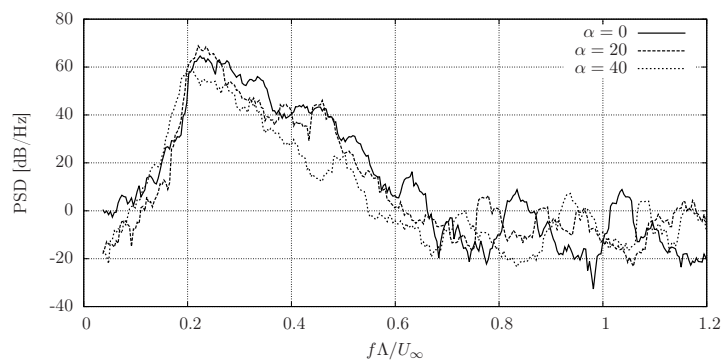

(c) ZTCBC

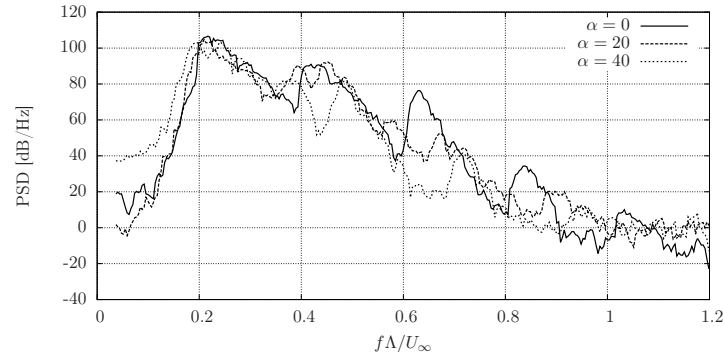

(b) ZODI

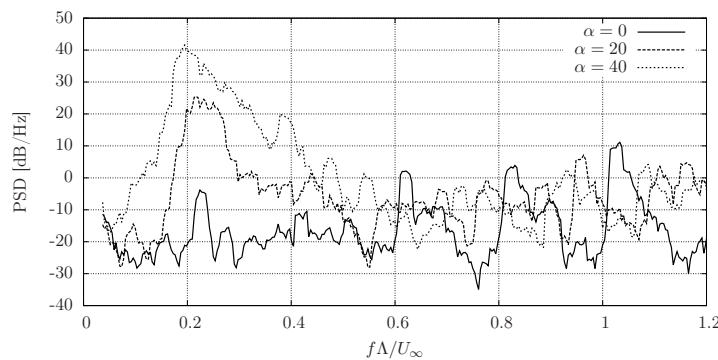

(d) ZTCBC-M

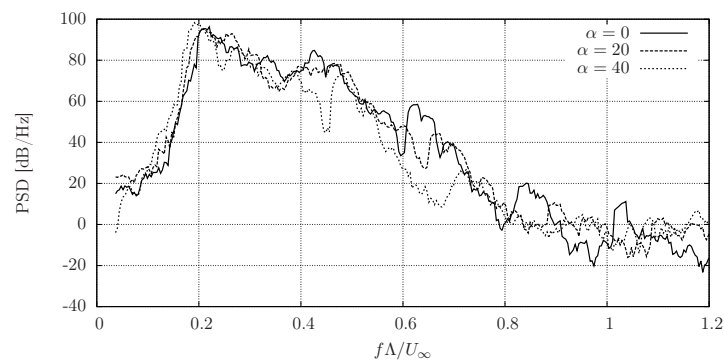

(e) ZTCBC-O

Figure 11. The acoustic response by synthesized turbulence impinging the outflow at various angles.

is used. The parameters $\phi$ and $d$ should be adjusted to ensure that the blending function is unity $\left(s_{f}=1\right)$ across the region of the outflow boundary dominated by vortical disturbances. The test case considered for the ZTCBC-B method employs the same grid and numerical methods described in Section IV.B, with a reduced injection plane size of $2.9 \mathrm{~m}<y<3.1 \mathrm{~m}$, to consider an outflow boundary with a localized region that is dominated by vortical disturbances. The integral length scale and the turbulent intensity are set to $\Lambda=0.25 \mathrm{~m}$ and $I_{x x}=0.01 U_{\infty}$, respectively.

The changes to the acoustic response spectrum with different values of $\phi$ are shown by Figure 12. At $M=0.2$ a larger $\phi$ generates a smaller acoustic response. As $\phi$ is reduced, the length of the outflow where the ZTCBC-M is used will decrease. This results in the vortical flow region being treated either by the ZTCBC-O method, or by a combination of both ZTCBC-O and ZTCBC-M (due to the continuous blending by the hyperbolic tangential function). This leads to an increased acoustic response that has a greater effect on the lower frequency range. As the Mach number is increased from $M=0.2$ to $M=0.5$, the effects of smaller $\phi$ become less pronounced. Therefore, the influence of $\phi$ is more important for low Mach number problems. As the Mach number is increased, the amplitude of the acoustic spectrum increases uniformly across the frequency range. However, this effect is also observed from the ZTCBC-M results in Figure 8.

The changes to the acoustic response by a ZTCBC-B method due to varying $d$ is shown in Figure 13. As $d$ is reduced, the maximum gradient of $s_{f}$ will increase and create a sharper switch. A smaller $d$ is favorable, as this reduces the size of the region in which a combination of the ZTCBC-M and ZTCBC-O methods is applied. As the size of this region is reduced the performance of the blended method is improved towards 


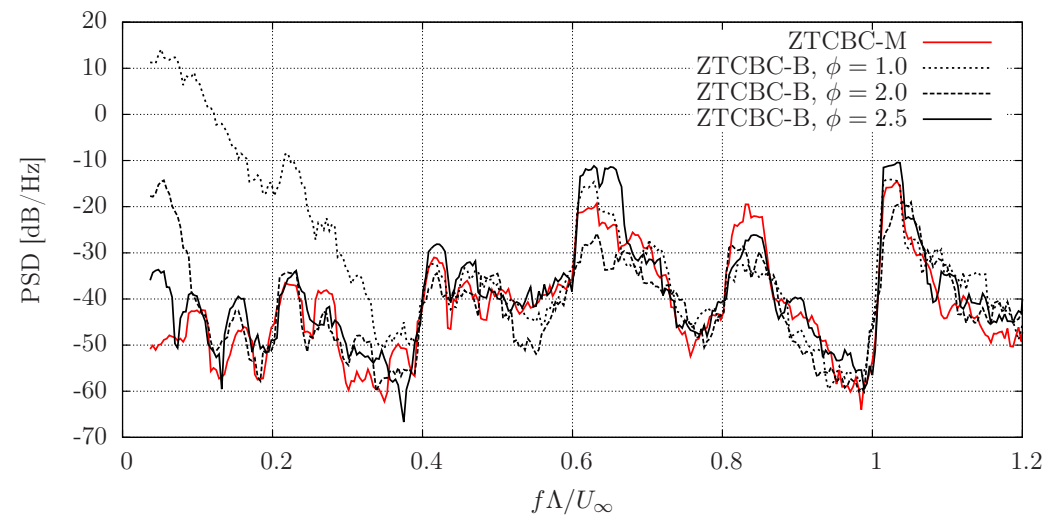

(a) $M=0.2$

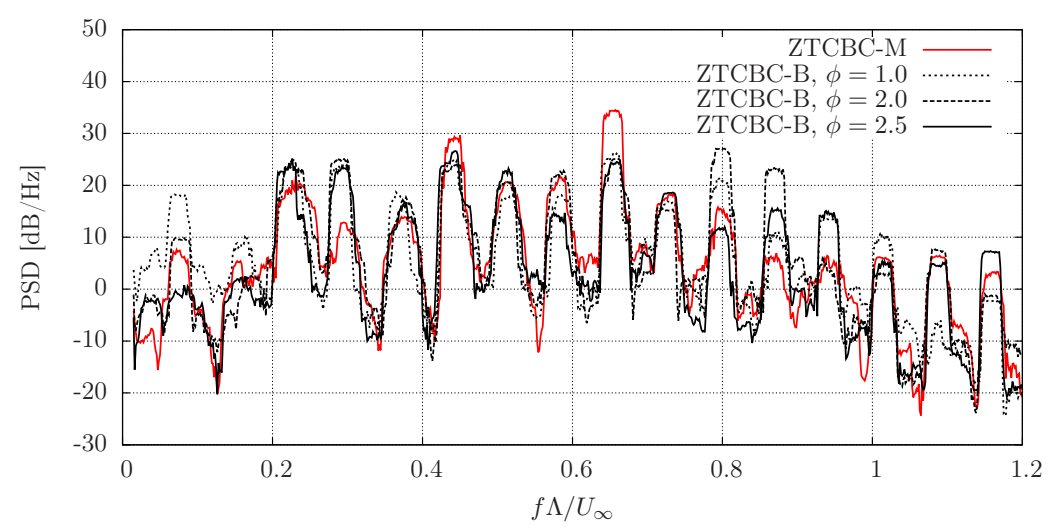

(b) $M=0.5$

Figure 12. The effect of $\phi$ on the acoustic response by the blended zonal characteristic outflow boundary condition, using $d=0.2$.

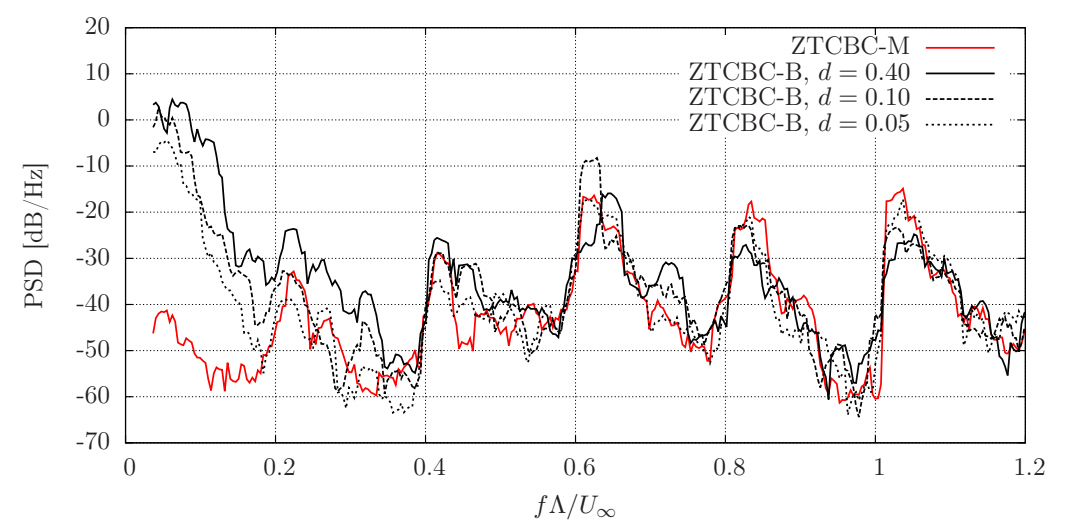

Figure 13. The effect of $d$ on the acoustic response by the blended zonal characteristic outflow boundary condition, using $\phi=1.5$. 
the lower frequency range.

The acoustic performance of the switching region can be predicted by data presented in Figure 14, which is taken from the work by Gill et al. ${ }^{6}$ In Figure 14 the ZTCBC-M refers to a condition where $A=1$ and $B=1$, and the ZTCBC-O refers to a point where $A=0.3$ and $B=0.7$. The blending function defined in this section only varies across the $y$-axis, and therefore, the $\mathrm{CBC}$ will not vary in the direction normal to the outflow plane. Figure 14 shows that the reflection generated at a fixed ratio of streamwise and transverse wave number (related to the wave angle), varies in a fairly linear manner between the points that define the ZTCBC-O and ZTCBC-M methods. Therefore, the performance of the ZTCBC-B method in the switching region is expected to fall between the performance of the ZTCBC-M and ZTCBC-O methods.
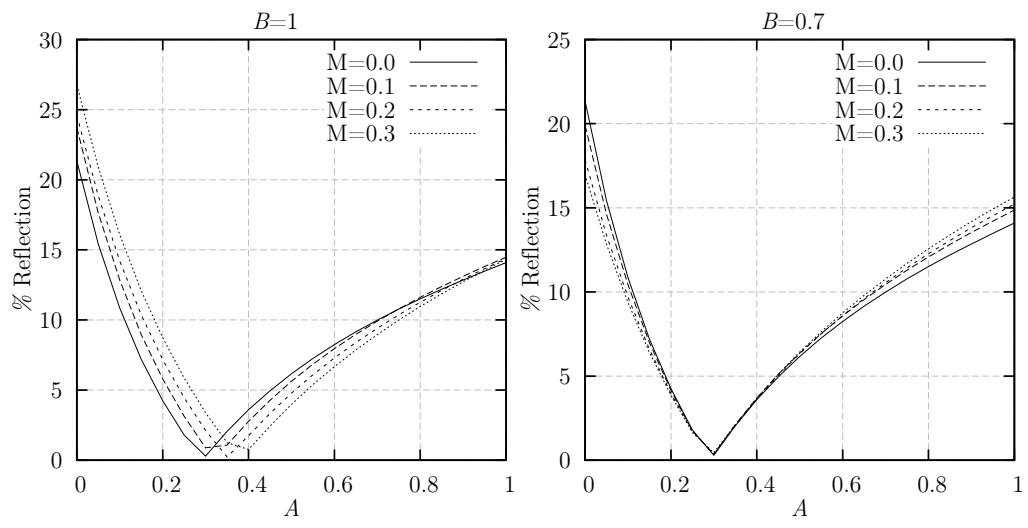

Figure 14. Acoustic reflections for waves with $\xi=1.1$ at various Mach numbers, and with various values of $A$. Results are shown for $B=1$ (left) and $B=0.7$ (right). ${ }^{6}$

The results in this section suggest that an appropriately defined blending function could define a generalized outflow boundary condition that applies the ZTCBC-O method by default, but switches to a ZTCBC-M method in the region dominated by strong vortical disturbances. The purpose of this work is to find a nonreflective condition that does not require user intervention between cases. Therefore, it is preferable that the switch between the ZTCBC-O and ZTCBC-M methods is automatic, and does not require user intervention. This method could be developed further to introduce a blending function that uses local flow-field information to automatically identify regions of the outflow that should be treated by the ZTCBC-M method.

\section{Conclusions}

In Computational Aeroacoustic (CAA) simulations, the need for non-reflective boundary conditions is imperative. These boundary conditions are applied along the outer regions of the computational domain, and are usually subjected to acoustic and vortical disturbances. Gill et al. ${ }^{6}$ recently compared the reflections generated by several non-reflective boundary conditions subjected only to acoustic disturbances. Vortical waves can be problematic as they may deform when they interact with an outflow boundary condition. This deformation may generate an upstream and downstream traveling acoustic wave. The latter may be partially reflected by the outflow boundary condition and will contribute to the overall contamination of the numerical solution.

The present work is an extension of the study by Gill et al.. This work is focused on the acoustic response due to vortical disturbances impinging on several outflow boundary conditions. Some acoustic plane wave studies are also included. Several types of Characteristic Boundary Conditions (CBCs) are evaluated in this work that test the implications of zonal characteristic forcing regions and of different inclusions of the transverse characteristic terms. Another common non-reflective boundary condition method is the implicit buffer-zone, which has a simple implementation and induces a relatively low acoustic reflection if the wave angle is less than 40 degrees. However, this method is shown here to perform poorly for vortical disturbances in comparison to characteristic-based methods.

The best non-reflective performance from acoustic waves is obtained by the ZTCBC-O method of Gill et $a l .{ }^{6}$ However, for vortical disturbances the best results are obtained by the ZTCBC-M method, which uses a different inclusion of the transverse characteristic terms. The ZTCBC-M method is sensitive to varying 
Mach number, but despite this it provides the smallest acoustic response for vortical waves interacting with the domain edges. As the size of the zonal CBC region is increased, the performance of the ZTCBC-O and ZTCBC-M methods will become similar. However, increasing the size of the zone will increase the computational cost and is therefore not recommended.

For compressible flow simulations, the outflow boundary face may be subjected to both outgoing vortical and acoustic disturbances. Different regions of the outflow face may be dominated by one type of disturbance, and are therefore best treated by either the ZTCBC-M or the ZTCBC-O methods. A blending method is proposed here that switches smoothly between the two ZTCBC methods, and this was found to perform well for a modified broadband turbulence test case. The blending function applies the ZTCBC-M method in regions dominated by vortical disturbances, and changes to the ZTCBC-O method where only acoustic waves are encountered. It has been found that the best performance is obtained with a sharp switch between the two methods in order to minimize areas where a combination of the two is applied. An extension of the blending function, based on the local vorticity magnitude or the amplitude of the characteristic waves carrying the vortical waves, will be considered to derive a generalized outflow boundary condition for acoustic and vortical disturbances.

\section{References}

${ }^{1}$ Tam, C. K. W. and Dong, Z., "Radiation and outflow boundary conditions for direct computation of acoustic and flow disturbances in a non-uniform mean flow," Journal of Computational Acoustics, Vol. 04, No. 02, 1996, pp. 175-201.

${ }^{2}$ Tam, C. K. W. and Webb, J. C., "Dispersion-relation-preserving finite difference schemes for computational acoustics," Journal of Computational Physics, Vol. 107, No. 2, 1993, pp. 262-281.

${ }^{3}$ Bogey, C. and Bailly, C., "Three dimensional non reflective boundary conditions for acoustic simulations - far field formulation and validation test cases," Acta Acustica United With Acoustica, Vol. 88, 2002, pp. 463-471.

${ }^{4}$ Freund, J. B., "Proposed Inflow/Outflow Boundary Condition for Direct Computation of Aerodynamic Sound," AIAA Journal, Vol. 35, No. 4, 1997, pp. 740-742.

${ }^{5}$ Richards, S., Zhang, X., Chen, X., and Nelson, P., "The evaluation of non-reflecting boundary conditions for duct acoustic computation," Journal of Sound and Vibration, Vol. 270, No. 3, 2004, pp. 539-557.

${ }^{6}$ Gill, J., Fattah, R., and Zhang, X., "Evaluation and development of non-reflective boundary conditions for aeroacoustic simulations," 21st AIAA/CEAS Aeroacoustics Conference, No. AIAA 2015-2677, 2015.

${ }^{7} \mathrm{Hu}$, F., Hussaini, M., and Manthey, J., "Low-dissipation and low-dispersion RungeKutta schemes for computational acoustics," Journal of Computational Physics, Vol. 124, No. 1, 1996, pp. 177-191.

${ }^{8}$ Fosso P., A., Deniau, H., Lamarque, N., and Poinsot, T., "Comparison of outflow boundary conditions for subsonic aeroacoustic simulations," International Journal for Numerical Methods in Fluids, Vol. 68, No. 3, 2012, pp. $1207-1233$.

${ }^{9}$ Thompson, K. W., "Time dependent boundary conditions for hyperbolic systems," Journal of Computational Physics, Vol. 68, No. 1, 1987, pp. 1-24.

${ }^{10}$ Thompson, K. W., "Time-dependent boundary conditions for hyperbolic systems, \{II $\}$," Journal of Computational Physics, Vol. 89, 1990, pp. 439-461.

${ }^{11}$ Poinsot, T. J. and Lele, S. K., "Boundary conditions for direct simulations of compressible viscous flows," Journal of Computational Physics, Vol. 101, No. 1, 1992, pp. 104-129.

${ }^{12}$ Yoo, C. S. and Im, H. G., "Characteristic boundary conditions for simulations of compressible reacting flows with multidimensional, viscous and reaction effects," Combustion Theory and Modelling, Vol. 11, No. 2, 2007, pp. 259-286.

${ }^{13} \mathrm{Liu}$, Q. and Vasilyev, O. V., "Nonreflecting boundary conditions based on nonlinear multidimensional characteristics," International Journal for Numerical Methods in Fluids, Vol. 62, No. February 2009, 2010, pp. 24-55.

${ }^{14}$ Sandberg, R. D. and Sandham, N. D., "Nonreflecting zonal characteristic boundary condition for direct numerical simulation of aerodynamic sound," AIAA Journal, Vol. 44, No. 2, 2006, pp. 402-405.

${ }^{15}$ Hixon, R., Shih, S. H., and Mankbadi, R. R., "Evaluation of boundary conditions for computational aeroacoustics," 33rd AIAA Aerospace Sciences Meeting, , No. AIAA 95-0160, 1995.

${ }^{16}$ Gea-Aguilera, F., Zhang, X., Chen, X., Gill, J., and Node-Langlois, T., "Synthetic turbulence methods for leading edge noise predictions," 21st AIAA/CEAS Aeroacoustics Conference, , No. AIAA-2015-2670, 2015.

${ }^{17}$ Liu, W., Kim, J. W., Zhang, X., Angland, D., and Caruelle, B., "Landing-gear noise prediction using high-order finite difference schemes," Journal of Sound and Vibration, Vol. 332, No. 14, 2013, pp. 3517-3534.

${ }^{18}$ Wang, M., Fattah, R., Angland, D., and Zhang, X., "High-order hybrid cell-centered method for computational aeroacoustics," 21st AIAA/CEAS Aeroacoustics Conference, No. AIAA 2015-3279, 2015.

${ }^{19} \mathrm{Kim}$, J. W. and Sandberg, R. D., "Efficient parallel computing with a compact finite difference scheme," Computers $8 \mathcal{6}$ Fluids, Vol. 58, No. 15 April 2012, 2012, pp. 70-87.

${ }^{20} \mathrm{Kim}$, J. W., "Optimised boundary compact finite difference schemes for computational aeroacoustics," Journal of Computational Physics, Vol. 225, No. 1, 2007, pp. 995-1019.

${ }^{21} \mathrm{Kim}, \mathrm{J} . \mathrm{W}$., "High-order compact filters with variable cut-off wavenumber and stable boundary treatment," Computers Es Fluids, Vol. 39, No. 7, 2010, pp. 1168-1182.

${ }^{22}$ Wilson, A., "A method for deriving tone noise information from CFD calculations on the aeroengine fan stage," RTO AVT Symposium on "Ageing Mechanisms and Control: Part A - Developments in Computational Aero- and Hyro-Acoustics", Vol. 079, No. October 2001, 2002, pp. 8-11. 
${ }^{23}$ Ewert, R., Appel, C., Dierke, J., and Herr, M., "RANS/CAA Based Prediction of NACA 0012 Broadband Trailing Edge Noise and Experimental Validation," 15th AIAA/CEAS Aeroacoustics Conference, , No. AIAA 2009-3269, 2009.

${ }^{24}$ Cozza, I. F., Iob, a., and Arina, R., "Broadband trailing-edge noise prediction with a stochastic source model," Computers and Fluids, Vol. 57, 2012, pp. 98-109.

${ }^{25}$ Kim, D., Lee, G.-S., and Cheong, C., "Inflow broadband noise from an isolated symmetric airfoil interacting with incident turbulence," Journal of Fluids and Structures, Vol. 55, 2015, pp. 428-450. 